\title{
A new tool in regenerative medicine: mesenchymal stem cell secretome
}

\begin{abstract}
Mesenchymal Stem Cells (MSCs) have been discovered and derived from numerous somatic tissue sources and have been extensively studied as a potential cellular therapy product in the realm of regenerative medicine. MSC cell-based therapy has been demonstrated to be safe and effective in several pre-clinical models encompassing numerous disease states. It is becoming apparent that the paracrine effect or the secretome of the MSCs has a major role in tissue repair. This tissue repair is mediated via trophic factors that; reduce tissue injury, recruit endogenous progenitor cells, positively influence angiogenesis, inflammation, cytoprotection, metabolic modulation and anti-apoptotic factors. In addition, several recent discoveries suggest that lipid microvesicles released by MSCs may also be important for the physiological function of these cells and also play a role in the paracrine effect. This current review will discuss the potential use of these tropic factors in the treatment of various diseases and other questions surrounding this type of therapy.
\end{abstract}

Keywords: mesenchymal stem cells (msc), paracrine effect, regenerative medicine, cell therapy, inflammation, apoptosis, endosomes, bone marrow, adipose tissue, dental pulp, wharton's jelly, microvesicles, RNA, parkinson's disease, alzheimer's disease, myocardial infarction, multipotent
Volume I Issue I - 2015

\section{Todd R Flower,Victoria Pulsipher,Andres Moreno}

Celebration Stem Cell Centre, USA

Correspondence: Todd R Flower, PhD, Celebration Stem Cell Centre, 3495 S. Mercy Road, Gilbert, AZ, USA, Tel 480722 9963, Fax 480722 9965, Email tflower@csccaz.com

Received: July 3, 2015 | Published: July 31, 2015
Abbreviations: MSC, mesenchymal stem cells; CMSC, cardiac mesenchymal stem cells; MV, micro vesicles; $\mathrm{CM}$, conditioned media; ISCT, international society for cellular therapy

\section{Introduction}

Mesenchymal Stem Cells (MSCs), often referred to as stromal cells have been the focus in regenerative medicine for their immune suppressive ability, their ability to hone in on the site of injury, their ability to differentiate into multiple cell lines, and ease of cell culture expansion. MSCs have the ability to differentiate into a range of specialized cells of mesodermal origin bone, cartilage, fat, cardio myocytes, muscle fibers, renal tubular cells), differentiate into cells of ectodermal origin (neurons) and cells of the endodermal origin (hepatocytes and pancreatic islands). Due to these properties MSCs are considered a new and exciting treatment option in the field of regenerative medicine.

The discovery of MSCs was originally reported by Friedenstein et al., ${ }^{1}$ in 1968 with the discovery that bone marrow contains a large number of stem cells, and Caplan et al., ${ }^{2}$ named them MSCs. ${ }^{1-3}$ Accumulating evidence shows that MSCs may be the most promising choice for research in tissue engineering and regenerative medicine because of their immune suppressive and anti-inflammatory properties. ${ }^{4,5}$ As a hallmark of any stem cell type MSCs possess the capabilities of self-renewal and differentiation into multiple cell lineages.

Given the absence of specific MSC markers and the observation that the morphology of these cells can vary, it can be challenging to easily identify these cells. Based on this The International Society for Cellular Therapy (ISCT) established the minimal requirements of the MSC: plastic adherence, positive expression of the cell surface markers CD73, CD90 and CD105, and negative expression for the hematopoietic markers CD34 and CD45. In addition, MSCs need to demonstrate the ability to differentiate into mesenchymal cell types including Adipocytes, chondrocytes and osteoblasts. ${ }^{6}$

In general MSCs are immature cells that can be found in numerous different tissue types, including but not limited to bone marrow, peripheral blood, menstrual blood, Adipose tissue, and the pulp chamber of teeth. ${ }^{7-9}$ MSCs derived from different tissue sources share many similarities, but also seem to have numerous differences with respect of their gene expression and their biological properties (e.g. differentiation potential). This suggests that MSCs from different tissue sources may work better in some regenerative medicine applications compared to others. It has been shown, for example, that MSCs from different tissue origins of the same donor differ in some key features. MSCs isolated from the bone-marrow and cardiac tissue (c MSCs) shared a common immunological phenotype, however, their gene micro RNA and protein expression profiles were remarkably different. CMSCS were less competent in acquiring the adipogenic and osteogenic phenotype, but showed a higher cardiovascular differentiation potential. ${ }^{10}$

\section{MSCs in regenerative medicine}

The unique characteristics of MSCs, such as their multipotentcy, immunological properties and effects on tissue repair, raised expectations on the possibility to use these cells for clinical applications in regenerative medicine. ${ }^{11}$ MSCs have been used to treat bone disorders, ${ }^{12}$ myocardial infarction, ${ }^{13}$ neurodegenerative diseases such as Parkinson's, Alzheimer's disease ${ }^{14-15}$ and kidney injury. ${ }^{16}$ Interestingly enough, it was determined that in the case of myocardial infarction only a small percentage of MSCs administered in swine are retained in the heart 2 weeks post-transplantation, and only 2.0 to $2.5 \%$ of MSCs engrafted in the kidney post-transplantation. This is surprising since both models demonstrated substantial tissue repair, 
which is indicative of a different mechanism of repair aside from integration and differentiation.

\section{MSC paracrine effect and MSC-released vesicles as a new tool for regenerative medicine}

MSCs appear to have beneficial effects with respect to repair of damaged tissue aside from their multi potent self-renewal potential and their ability to trans differentiate into multiple germ layers. Based on the experimental data above and other data demonstrating that only a small percentage of MSCs will actually be integrated into the host damaged tissue ${ }^{17,18}$ a strong inference can be made that tissue repair is an indirect effect possibly based on the paracrine effect of the MSC as opposed to MSC engraftment.

MSC-secreted molecules may act as paracrine mediators that directly activate target cells or cause neighboring cells to secrete bioactive agents. ${ }^{19}$ It has also recently been demonstrated that extracellular micro vesicles (MVs) released from cells are integral for the cell-cell communication network involved in tissue regeneration..$^{20,21}$ Additional support for the paracrine action of MSCs is provided by experiments demonstrating that MSC-Conditioned Media (CM) mimics the reparative effects of the MSCs themselves. ${ }^{22}$

MSC-secreted micro vesicles, or exosomes arise from the endosomal membrane cell compartment and are released into the extracellular space after fusion of multi vesicular bodies with the plasma membrane..$^{23}$ They tend to be similar in size $(30-120 \mathrm{nM})$ and are released by p53-mediated exocytosis. Exosomes appear to contain a variety of different proteins and molecules. Despite the excitement and interest raised by MSC-derived micro vesicles for their potential role in regenerative medicine, only a limited number of studies have been conducted trying to identify the specific RNA and protein content of these vesicles. It is speculated that and at least some 800 other proteins, RNA molecules, growth factors, and cytokines might be secreted.

These MVs may interact with target cells through specific ligandreceptor interactions and transfer receptors and other molecules after the MVs are internalized. ${ }^{21}$ In addition, the MVs could carry different RNAs that could potentially play a role in the exchange of genetic information from cell-to-cell. ${ }^{24,25} \mathrm{MVs}$, through a wide variety of different mechanisms may influence the behavior of the target cells, which is outside the scope of this review. But briefly, by transferring selected molecules to target cells, the paracrine factors can act as potent signals between stem cells and differentiated cells.

\section{Discussion}

This emerging new facet of MSCs-that is, to facilitate the repair of a damaged tissue by using the paracrine factors in place of whole cells-introduces a very different dimension to the therapeutic use of MSCs in regenerative medicine. Even though MSCs, up until this point are considered safe, the development of therapeutic mechanisms that avoid the use of living stem cells will certainly have an effect on the attenuation on the safety concerns relative to cell origin and immune compatibility issues. A cell-free based approach could also minimize the biological variability, allow for more accurate dosing and therefore lead to safe and effective therapies with a more predictable outcome.

On the other hand there still several key issues that need to bestudied further. First, all of the components of the MSC secretome should be identified using current proteomic and metabolomics techniques before moving forward with therapeutic strategies. This will give us a better understanding of what we are working with and what the clinical outcomes might be. Second, the secretome may vary from MSCs harvested from different tissue sources. If this is the case then the MSC secretome from one tissue source might not be suitable to treat certain conditions, but suitable to treat others. Therefore, the characterization of secretomes from each common source of MSCs (Bone Marrow, Adipose Tissue, Dental Pulp, Wharton's Jelly, etc.) is required to develop the most effective therapies for each patient's needs.

Other factors that need consideration are the limitations of this type of therapy. Some key limitations are mostly due to tissue transport, pharmacokinetics, and protein stability, which stress the need to identify all the components of the secretome and develop novel release and delivery methods.

\section{Conclusions}

Regenerative medicine is a subject of great expectations and his new facet of regenerative medicine, i.e. the paracrine effect of MSCs, has changed the perspective of the use of MSCs in a therapeutic setting with respect to using a cell-free therapeutic system. This can potentially alleviate many safety concerns with respect to using live cells.

Uncovering the molecular and biochemical pathways mediating the MSC paracrine effect is essential toward our understanding of the secreted protein profile and its potential clinical use. ${ }^{26}$ In addition to proteomic approaches, studies are now being conducted to utilize the next-generation metabolomics-driven approaches coupled with biological systems to uncover key metabolic pathways mediating the MSC paracrine effect. Understanding these mechanisms may prove to be an essential step to maximize therapeutic potential of MSC paracrine effect in regenerative medicine.

Beyond the great enthusiasm for potential new treatments, much research is needed on the development of safe and effective MSC paracrine therapies, which will lay the foundation for new regenerative medicine approaches (Figure 1).

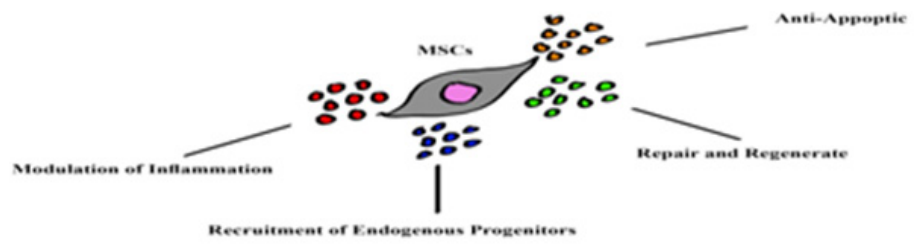

Figure I Paracrine Effect of Mesenchymal Stem Cells.

\section{Acknowledgements}

Funding and support for all work is provided by Bill and Sherry Lund.

\section{Conflict of interest}

The author declares no conflict of interest.

\section{References}

1. Friedenstein AJ, Petrakova KV, Kurolesova AI, et al. Heterotopic transplants of bone marrow. Analysis of precursor cells for osteogenic and hematopoietic tissues. Transplantation. 1968;6(2):230-247. 
2. Caplan A, Bruder SP. Mesenchymal stem cells: building blocks for molecular medicine in the 21st century. Trends Mol Med. 2001;7(6):259264.

3. Caplan A. Mesenchymal stem cells. J Orthop Res. 1991;9(5):641-650.

4. Ankrum J, Karp JM. Mesenchymal stem cell therapy: Two steps forward, one step back. Trends Mol Med. 2010;16(5):203-209.

5. Phinney DG, Prockop DJ. Concise review: mesenchymal stem/multipotent stromal cells: the state of transdifferentiation and modes of tissue repair-current views. Stem Cells. 2007;25(11):2896-2902.

6. Dominici M, Le Blanc K, Mueller, et al. Minimal criteria for defining multipotent mesenchymal stromal cells. The International Society for Cellular Therapy position statement. Cytotherapy. 2006;8(4):315-317.

7. da Silva Meirelles L, Chagastelles PC, Nardi NB. Mesenchymal stem cells reside in virtually all post-natal organs and tissues. J Cell Sci. 2006;119(11):2204-2213.

8. Kassis I, Zangi L, Rivkin R, et al. Isolation of mesenchymal stem ce1ls from G-CSF-mobilized human peripheral blood using fibrin microbeads. Bone Marrow Transplant. 2006;37(10):967-976.

9. Zou Z, Zhang Y, Hao L, et al. More insight into mesenchymal stem cells and their effects inside the body. Expert Opin Biol Ther. 2010;10(2):215230

10. Rossini A, Frati C, Lagrasta C, et al. Human cardiac and bone marrow stromal cells exhibit distinctive properties related to their origin. Cardiovasc Res. 2011;89(3):650-660.

11. Lee RH, Kim B, Choi I, et al. Characterization and expression analysis of mesenchymal stem cells from human bone marrow and adipose tissue. Cell Physiol Biochem. 2004;14(4-6):311-324.

12. Horwitz EM, Gordon PL, Koo WK, et al. Isolated allogeneic bone marrow-derived mesenchymal cells engraft and stimulate growth in children with osteogenesis imperfecta: implications for cell therapy of bone. Proc Natl Acad Sci U S A. 2002;99(13) 8932-8937.

13. Toma C, Pittenger MF, Cahill KS, et al. Human mesenchymal stem cells differentiate to a cardiomyocyte phenotype in the adult murine heart. Circulation. 2002;105(1):93-98.

14. Trzaska KA, Rameshwar P. Dopaminergic neuronal differentiation protocol for human mesenchymal stem cells. Methods Mol Biol. 2011;698:295-303.
15. Lee JK, Jin HK, Endo S, et al. Intracerebral transplantation of bone marrow-derived mesenchymal stem cells reduces amyloid-beta deposition and rescues memory deficits in Alzheimer's disease mice by modulation of immune responses. Stem Cells. 2010;28(2):329-343.

16. Morigi M, Imberti B, Zoja C, et al. Mesenchymal stem cells are renotropic, helping to repair the kidney and improve function in acute renal failure. J Am Soc Nephrol. 2004;15(7):1794-1804.

17. Rosario CM, Yandava BD, Kosaras B, et al. Differentiation of engrafted multipotent neural progenitors towards replacement of missing granule neurons in meander tail cerebellum may help determine the locus of mutant gene action. Development. 1997;124(21): 4213-4224.

18. Li TS, Takahashi M, Ohshima M, et al. Myocardial repair achieved by the intramyocardial implantation of adult cardiomyocytes in combination with bone marrow cells. Cell Transplant. 2008;17(6):695-703.

19. Caplan A, Dennis JE. Mesenchymal stem cells as trophic mediators. $J$ Cell Biochem. 2006;98(5):1076-1084.

20. Ratajczak J, Miekus K, Kucia M, et al. Embryonic stem cell-derived microvesicles reprogram hematopoietic progenitors: evidence for horizontal transfer of mRNA and protein delivery. Leukemia. 2006;20(5):847856.

21. Camussi G, Deregibus MC, Bruno S, et al. Exosomes/microvesicles as a mechanism of cell-to-cell communication. Kidney Int. 2010;78(9):838848 .

22. Bi B, Schmitt R, Israilova M, et al. Stromal cells protect against acute tubular injury via an endocrine effect. J Am Soc Nephrol. 2007;18(9):24862496.

23. Mathivanan S, Ji H, Simpson RJ. Exosomes: extracellular organelles important in intercellular communication. J Proteomics. 2010;73(10):19071920.

24. Deregibus MC, Cantaluppi V, Calogero R, et al. Endothelial progenitor cell derived microvesicles activate an angiogenic program in endothelial cells by a horizontal transfer of mRNA. Blood. 2007;110(7):2440-2448.

25. Yuan A, Farber EL, Rapoport AL, et al. Transfer of microRNAs by embryonic stem cell microvesicles. PLoS One. 2009;4(3):e4722.

26. Karamouzian S, Nematollahi-Mahani SN, Nakhaee N, et al. Clinical safety and primary efficacy of bone marrow mesenchymal cell transplantation in subacute spinal cord injured patients. Clin Neurol Neurosurg. 2012;114(7):935-939. 